\title{
Smoking Behaviour before, during, and after Pregnancy: The Effect of Breastfeeding
}

\author{
Laura Lauria, Anna Lamberti, and Michele Grandolfo \\ National Center of Epidemiology, Surveillance and Health Promotion, National Institute of Health, 00161 Rome, Italy \\ Correspondence should be addressed to Laura Lauria, laura.lauria@iss.it
}

Received 20 October 2011; Accepted 17 November 2011

Academic Editors: S. H. Opdal and M. J. Zvolensky

Copyright () 2012 Laura Lauria et al. This is an open access article distributed under the Creative Commons Attribution License, which permits unrestricted use, distribution, and reproduction in any medium, provided the original work is properly cited.

Data for this study were obtained from a population-based follow-up study in 25 Italian Local Health Units (LHUs) to evaluate pregnancy, delivery, and postpartum care in Italy. A sample of 3534 women was recruited and interviewed within a few days of their giving birth and at 3,6, and 12 months after delivery, by trained interviewers using questionnaires. The objective of the study was to evaluate changes in smoking behaviour from one interview to the next. Of 2546 women who completed the follow-up, smoking prevalences before and during pregnancy were $21.6 \%$ and $6.7 \%$; smoking prevalences and smoking relapse at 3, 6, and 12 months were $8.1 \%$ and $18.5 \%, 10.3 \%$ and $30.3 \%$, and $10.9 \%$ and $32.3 \%$, respectively. Smoking during and after pregnancy was more likely among women who were less educated, single, not attending antenatal classes, employed, and not breastfeeding. The results show that women who are breastfeeding smoke less than not breastfeeding women, even after controlling for other predictors (i.e., smoking relapse at 12 months: $\mathrm{OR}=0.43,95 \% \mathrm{CI}$ : 0.19, 0.94). A low maternal mood increases the risk of smoking relapse within 6 months of about $73 \%$. This study also suggests that prolonged breastfeeding reduces the risk of smoking relapse and that this reduction may be persistent in time. Interventions targeting breastfeeding promotion may also indirectly support smoking cessation, even in absence of specific interventions.

\section{Introduction}

Smoking is one of the most important avoidable causes of disability, mortality, and adverse maternal and fetal outcomes in Western countries $[1,2]$.

During recent decades, researchers have studied the adverse effects of smoking on conception, pregnancy, fetal, and child health $[3,4]$. The associated adverse outcomes include low birth weight, reduced fetal growth, placenta previa, preterm birth, respiratory infections, asthma, sudden infant death syndrome [3-6], and hyperkinetic disorders [7].

In recent years, almost all Western countries have registered a decrease in smoking prevalence in the general population and in particular among pregnant women. Data from the Pregnancy Risk Assessment Monitoring System (PRAMS) show a slight reduction in smoking prevalence during pregnancy, in the period $2000-2005$, from $15.2 \%$ to $13.8 \%$ [2]. However, these values remain far from the goal of $2 \%$ during pregnancy given by "Healthy People-2010" [8]. After a decreasing trend, the prevalences of smoking in pregnancy were 11\% in Canada in 2005 [9], 16\% in Denmark in 2005 (among pregnant nulliparous women) [10], and 20\% in Australia in 2004 [11]. An opposite trend was registered in Japan where the prevalence of smoking in pregnancy increased from $5.6 \%$ in 1991 to $10 \%$ in 2001 [12]. Moreover, an increasing trend has been forecast for Eastern Europe and Asiatic countries [2]. According to the last European report on perinatal health, more than $10 \%$ of women smoke during pregnancy with values ranging from $5 \%$ in Lituania to $22 \%$ in France [13].

In Italy, the prevalence of smoking during pregnancy declined from $9.2 \%$ in 2000 to $6.5 \%$ in 2005 . The percentage of smokers who quit during pregnancy increased from $63.4 \%$ in 2000 to $70.7 \%$ in 2005 ; it has also been estimated that almost one third of quitters do so permanently [14]. Nevertheless, more recent surveys show that in 2009 there was an increase in the prevalence of smoking in the general population and specifically in women of reproductive age $[15,16]$. 
Several studies have shown that pregnancy is a period when many smokers quit, although most of them relapse within one year of delivery. About $40 \%$ of smokers quit during pregnancy, but of them, $45 \%$ relapse within 3 months from delivery, 60-70\% within 6 months, and almost $80 \%$ within one year [17]. In order to plan effective health promotion interventions, several studies have focused on the factors which predict quitting smoking during pregnancy and relapsing postpartum. Factors considered as potential predictors of the smoking behaviour change during and after pregnancy are socioeconomic status, education, stress, living with a smoker, marital status, age, parity, employment, and breastfeeding $[2,18]$. Breastfeeding is one of the strongest factors associated with smoking abstinence after pregnancy [19-21]. Nevertheless, the nature of the relationship between breastfeeding and smoking needs further research. It is not clear if breastfeeding promotes smoking cessation or smoking cessation prolongs breastfeeding or, alternatively, if there is a third unidentified factor such as stress or family/social support, which may influence both breastfeeding and smoking behaviour [21-23]. In this study we investigated the effect of maternal mood and participation in antenatal classes (ACs).

The aims of this study are (i) to explore the prevalences of smoking before, during, and at 3, 6, and 12 months after pregnancy, (ii) to investigate the factors associated with smoking cessation during pregnancy and smoking relapse after pregnancy, and (iii) to focus on the relationship between breastfeeding and smoking relapse.

\section{Materials and Methods}

Data for this study were obtained from a populationbased follow-up study of maternal behaviors and maternal health-care services during preconception, pregnancy, and postpartum conducted by the Italian Institute of Public Health in 2009 [24]. The survey was offered to all of the 20 Italian administrative Regions, but only 11 agreed to participate. Each region is divided into administrative Local Health Units (LHUs). Twenty-five of the 79 LHUs present in the participating Regions, participated in the study. Women who had given birth and resident in these LHUs were the target population (about 45000 new births each year). Both town and hospital registers of births were used as sampling frame.

A pseudo-random sampling method was used to recruit 120 women who had given birth in each LHU. All women who had given birth were recruited until 120 were reached in each LHU. Exclusion criteria were as follows: severe illness of mother or child; women with an active infection and fever $>38^{\circ}$, and women with hematic loss $>1000 \mathrm{cc}$.

In order to make the entire sample representative of the total population from which the LHU samples were derived, descriptive and multivariate analyses were weighted by the reciprocal of the sampling fractions. Women were interviewed face to face $(91 \%)$ or by phone $(9 \%)$ by trained interviewers using a questionnaire, preferably in hospital after delivery or within a few days from discharge.
The reinterviews of the same women were conducted by phone after three, six, and twelve months from delivery. At the end of each interview, the women were asked to give their consent to be reinterviewed at the successive follow-up date. The first questionnaire was structured in four sections regarding pregnancy, delivery, postpartum, and sociodemographic characteristics. For multiparous women, items regarding breastfeeding of the previous children were also included. The follow-up questionnaires included items regarding extra hospital assistance, breastfeeding, smoking behaviour, and a set of items aimed at defining a general maternal mood. The basic characteristics of women lost to follow-up were compared with those of women who completed the follow-up by the Pearson $\chi^{2}$ test. Smoking behaviour was the main outcome variable of this study. Prevalences of smoking were estimated as the ratio between the number of women who reported to be smoker at each point interview and the total number of interviewed women. Descriptive analyses on prevalence of smoking, on quitting smoking, and on smoking relapse within 3, 6, and 12 months from partum were reported. Weighted multivariate logistic models which take account of complex survey data were used to analyse the association between breastfeeding and smoking status after controlling for potential confounding factors. If not otherwise indicated, the analyses focused on women who completed the follow-up and were conducted using the statistical software STATA, version 11.

\section{Definition of Variables}

At delivery the women were asked two questions about their smoking behaviour: if they smoked regularly before pregnancy and if yes, if they also smoked during pregnancy. At 3, 6, and 12 months after delivery, the women were asked if they smoked regularly. Possible answers for all the questions on smoking behaviour were no or yes with the number of cigarettes per day specified. On the base of the number of cigarettes smoked before pregnancy, women were classified as light smokers ( $\leq 10$ cigarettes/day) or heavy smokers $(>10$ cigarettes/day).

Two different definitions of breastfeeding were used: "full breastfeeding" when children were exclusively breastfed or breastfed with addition of water/herbal teas (no formula was used), and "any breastfeeding" for any other addition to maternal milk. "Full breastfeeding" was used to predict smoking relapse at 3-month follow-up; "any breastfeeding" was considered at the 6- and 12-month follow-ups. Education was classified as low (less than high school), medium (high school), and high (university). Participation in antenatal classes (yes, no), consisting of a variable number of meetings of women and maternal/child care professionals during the present pregnancy and in puerperium, was also investigated. Employment status at each follow-up time was classified as not employed, employed who had restarted working after pregnancy, and employed who had not yet restarted working. A measure of "maternal mood" at each follow-up interview was created by combining the answers to 7 questions which compared life conditions after delivery 
TABLE 1: Sample characteristics.

\begin{tabular}{|c|c|c|c|c|c|}
\hline \multirow[t]{2}{*}{ Variables } & \multicolumn{2}{|c|}{$\begin{array}{c}\text { Completed follow-up } \\
\text { sample } \\
N=2546\end{array}$} & \multicolumn{2}{|c|}{$\begin{array}{l}\text { Lost to follow-up } \\
\text { sample } \\
N=988\end{array}$} & \multirow{2}{*}{$\begin{array}{l}\chi^{2} \\
P\end{array}$} \\
\hline & $N$ & Weighted \% & $N$ & Weighted \% & \\
\hline \multicolumn{6}{|l|}{ Age } \\
\hline$<30$ & 727 & 28.0 & 369 & 37.2 & 0.005 \\
\hline $30-34$ & 987 & 39.6 & 336 & 34.6 & \\
\hline$>35$ & 832 & 32.4 & 283 & 28.2 & \\
\hline \multicolumn{6}{|l|}{ Education } \\
\hline Low & 743 & 27.4 & 337 & 34.4 & $<0.001$ \\
\hline Medium & 1267 & 48.8 & 467 & 46.2 & \\
\hline High & 536 & 23.8 & 184 & 19.4 & \\
\hline \multicolumn{6}{|l|}{ Marital status } \\
\hline Married & 2015 & 77.6 & 738 & 73.2 & 0.100 \\
\hline Single/Sep/Div/Wid & 531 & 22.4 & 250 & 26.8 & \\
\hline \multicolumn{6}{|l|}{ Employment } \\
\hline No & 776 & 28.2 & 376 & 36.3 & 0.003 \\
\hline Yes & 1770 & 71.8 & 612 & 63.7 & \\
\hline \multicolumn{6}{|l|}{ Parity } \\
\hline Primiparous & 1368 & 55.5 & 477 & 49.8 & 0.036 \\
\hline Multiparous & 1144 & 44.5 & 483 & 50.2 & \\
\hline $\begin{array}{l}\text { Multiparous not breastfeeding previous birth } \\
\text { or breastfeeding less than } 3 \text { months }\end{array}$ & 387 & 35.4 & 174 & 39.5 & 0.268 \\
\hline Multiparous breastfeeding previous infant more than 3 months & 754 & 64.6 & 304 & 60.5 & \\
\hline \multicolumn{6}{|l|}{ Participation in antenatal classes } \\
\hline No & 1535 & 59.3 & 727 & 76.3 & $<0.001$ \\
\hline Yes & 995 & 40.7 & 253 & 23.7 & \\
\hline \multicolumn{6}{|l|}{ Smoking behaviour before pregnancy } \\
\hline No & 1964 & 78.4 & 743 & 75.2 & 0.335 \\
\hline Yes & 582 & 21.6 & 245 & 24.8 & \\
\hline Quit during pregnancy & 397 & 68.7 & 161 & 67.1 & 0.651 \\
\hline Smoking during pregnancy & 183 & 31.3 & 84 & 33.0 & \\
\hline \multicolumn{6}{|l|}{ Complete breastfeeding at discharge } \\
\hline No & 669 & 29.6 & 310 & 32.5 & 0.188 \\
\hline Yes & 1837 & 70.4 & 659 & 67.5 & \\
\hline
\end{tabular}

with those before conception. The first item required women to indicate: if their life was worse, improved, or not changed; the next four items required women to indicate if they felt more, less, or as before baby arrived with regard to (1) serenity, (2) tiredness, (3) whether they were "understood", (4) whether they were "supported", (5) the time they had for themselves, and (6) the attention paid to them. Women were considered in an uncomfortable status (low mood) if a worsening was indicated in 3 or more of the items. Maternal age at delivery was categorized in two classes, $\leq 30$ years and $>30$ years. Marital status was categorized as married and unmarried at the time of delivery; the latter category included women who were single, divorced, separated or widowed. Parity was categorized as primiparous and multiparous. In a subgroup analysis, multiparous women were categorized as multiparous who breastfed the previous child for less than 3 months and multiparous who breastfed the previous child for more than 3 months.

\section{Results}

A total of 3669 women met the eligibility criteria; 135 women were declined or were not found. The $96.3 \%$ (3534) of eligible women were recruited and interviewed at delivery; 2546 (72\%) were reinterviewed at 3, 6 and 12 months (Table 1). Women who completed the follow-up were not statistically different from women lost to follow-up with respect to the smoking behaviour before (21.6\% versus $24.8 \%$ ) and during pregnancy $(31.3 \%$ versus $33.0 \%)$, complete breastfeeding after delivery $(70.4 \%$ versus $67.5 \%)$, and marital status 
TABLE 2: Smoking behaviour description before, during, and after pregnancy.

\begin{tabular}{|c|c|c|c|c|c|}
\hline \multirow[t]{2}{*}{ No. of cigarettes } & \multirow{2}{*}{$\begin{array}{c}\text { Smoking before } \\
\text { pregnancy } \\
n\end{array}$} & \multirow{2}{*}{$\begin{array}{c}\text { Quit during } \\
\text { pregnancy } \\
N \text { (weighted \%) }\end{array}$} & \multicolumn{3}{|c|}{ Weighted $\%$ relapse (\% relapse among breastfeeding women) } \\
\hline & & & within 3 months & within 6 months & within 12 months \\
\hline$\leq 5$ & 209 & $173(84.7)$ & $11.5(4.0)$ & $21.2(6.5)$ & $24.5(11.8)$ \\
\hline $6-10$ & 187 & $127(65.2)$ & $21.5(12.4)$ & $33.6(16.9)$ & $33.8(25.3)$ \\
\hline$>10$ & 184 & $97(55.2)$ & $26.6(15.3)$ & $41.5(23.3)$ & $43.3(27.7)$ \\
\hline Total & 580 & $397(68.7)$ & $18.5(9.5)$ & $30.3(14.3)$ & $32.3(21.1)$ \\
\hline
\end{tabular}

(77.6\% versus $73.2 \%$ of married women); they were more likely to be primiparous ( $55.5 \%$ versus $49.8 \%$ ), older (>35 years: $32.4 \%$ versus $28.2 \%$ ), highly educated (23.8\% versus $19.4 \%)$, employed (71.8\% versus $63.7 \%)$, and participating in the $\mathrm{AC}$ ( $40.7 \%$ versus $23.7 \%$ ).

The following analysis focuses on the women who completed the follow-up. Most of them were older than 29 years $(72.0 \%)$, of medium/high education $(72.6 \%)$, married $(77.6 \%)$, employed $(71.8 \%)$, primiparous $(55.5 \%)$, not smokers (78.4\%), and not partecipating in AC (59.3\%).

The prevalence of smoking was $21.6 \%$ before and 6.7\% during pregnancy. At 3-, 6-, and 12-month followup, prevalence (prevalence among breastfeeding) was $8.1 \%$ (5.2\%), 10.3\% (5.0\%), and 10.9\% (6.6\%), respectively (data not reported in table).

In Table 2, the changes in smoking behaviour at different points in time, controlling for the number of smoked cigarettes before pregnancy, are reported. Among 580 smokers before pregnancy, $68.7 \%$ reported that they quit smoking during pregnancy. Light smokers were more likely than heavy smokers to quit smoking during pregnancy. Of the $183(31.3 \%)$ smokers who reported that they continued to smoke during pregnancy, about $66 \%$ (113) reported that they reduced their consumption of cigarettes.

Among the 397 women quitting smoking during pregnancy, $18.5 \%$ (9.5\% in case of "any breastfeeding") relapsed within 3 months, 30.3\% (14.3\% in case of "any breastfeeding") relapsed within 6 months, and 32.3\% (21.1\% in case of "any breastfeeding") relapsed within 12 months. Percentages of smoking relapse appear higher for heavier smokers.

In Table 3, the results of the logistic regression models for smoking prevalence before pregnancy, quitting smoking during pregnancy, and relapse smoking at 12-month followup are reported. Tobacco use before pregnancy was more likely for unmarried with respect to married women $(\mathrm{OR}=$ 2.30, 95\% CI: $1.75,3.02)$. Protective factors were medium and high education with respect to low (medium $\mathrm{OR}=0.65$, 95\% CI: 0.48, 0.89; high OR $=0.50,95 \% \mathrm{CI}: 0.37,0.67)$. The same regression model was also used for a subgroup analysis of pluriparous women $(n=1619)$ who were categorised with respect to the type of breastfeeding of their previous child. In comparison with pluriparous who did not breastfeed or breastfed their previous child for less than three months, tobacco use was less likely for pluriparous women who have breastfed previous child for more than 3 months ( $\mathrm{OR}=0.58$, 95\% CI: $0.42,0.80$ ) (not reported in table).
To quit smoking during pregnancy was more likely for medium and highly educated women than less educated women (medium education $\mathrm{OR}=1.76,95 \% \mathrm{CI}$ : 0.93, 3.32; high education $\mathrm{OR}=2.42,95 \% \mathrm{CI}: 0.94,6.27)$. Quitting was less likely for unmarried women $(\mathrm{OR}=0.38,95 \% \mathrm{CI}: 0.21$, $0.71)$, for heavy smokers $(\mathrm{OR}=0.45,95 \% \mathrm{CI}: 0.31,0.64)$, and for multiparous women $(\mathrm{OR}=0.61,95 \% \mathrm{CI}$ : 0.39, 0.96).

Among the 397 women quitting smoking during pregnancy, restarting at 12 months of follow-up, was positively associated with having been a heavy smoker $(\mathrm{OR}=2.38$, 95\% CI: $1.44,3.91)$; protective factors were participation in $\mathrm{AC}(\mathrm{OR}=0.43,95 \% \mathrm{CI}: 0.19,0.96)$ and any breastfeeding at follow-up time $(\mathrm{OR}=0.43,95 \% \mathrm{CI}$ : 0.19, 0.94). Women who were employed before pregnancy, and especially women who had not yet restarted working at the follow-up time, were less likely to relapse compared with women not employed (restart working OR $=0.47,95 \% \mathrm{CI}$ : 0.21, 1.06; not restart working $\mathrm{OR}=0.36,95 \% \mathrm{CI}: 0.09,1.36)$.

Logistic regression models were also used to analyze smoking relapse within 3-, 6-, and 12-month follow-up considering all the women interviewed at each follow-up point without the restriction on women completing the follow-up at 12 months and including the potential predictor "maternal mood" (Table 4). The effect of breastfeeding was statistically significant in the all three points but higher at 3 and 6 months in comparison to 12 months. The variable "maternal mood" shows also a statistically significant effect on smoking relapse at 3 months (low mood OR $=1.73$, 95\% CI: $1.21,2.47)$ and at 6 months (low mood OR = $1.73,95 \%$ CI: $1.03,2.90$ ), but not at 12 months (low mood $\mathrm{OR}=0.71,95 \% \mathrm{CI}: 0.46,1.10)$. We explored a potential interaction effect between breastfeeding and maternal mood at each follow-up time but no statistically significant effect was found.

\section{Discussion}

This population-based survey gave us the opportunity to investigate the prevalences and the changes in smoking behaviour of women who have given birth, from before conception to 1 year after delivery. The prevalence of smoking before pregnancy $(21.6 \%)$ was similar to that estimated by the Italian National Institute of Statistics (ISTAT) [14] in 2005 (20.1\% for women aged 25-34 years and 21.7\% for women aged 35-44 years). Analogously, similar values of smoking prevalence during pregnancy (6.7\%) (ISTAT 6.5\%) 


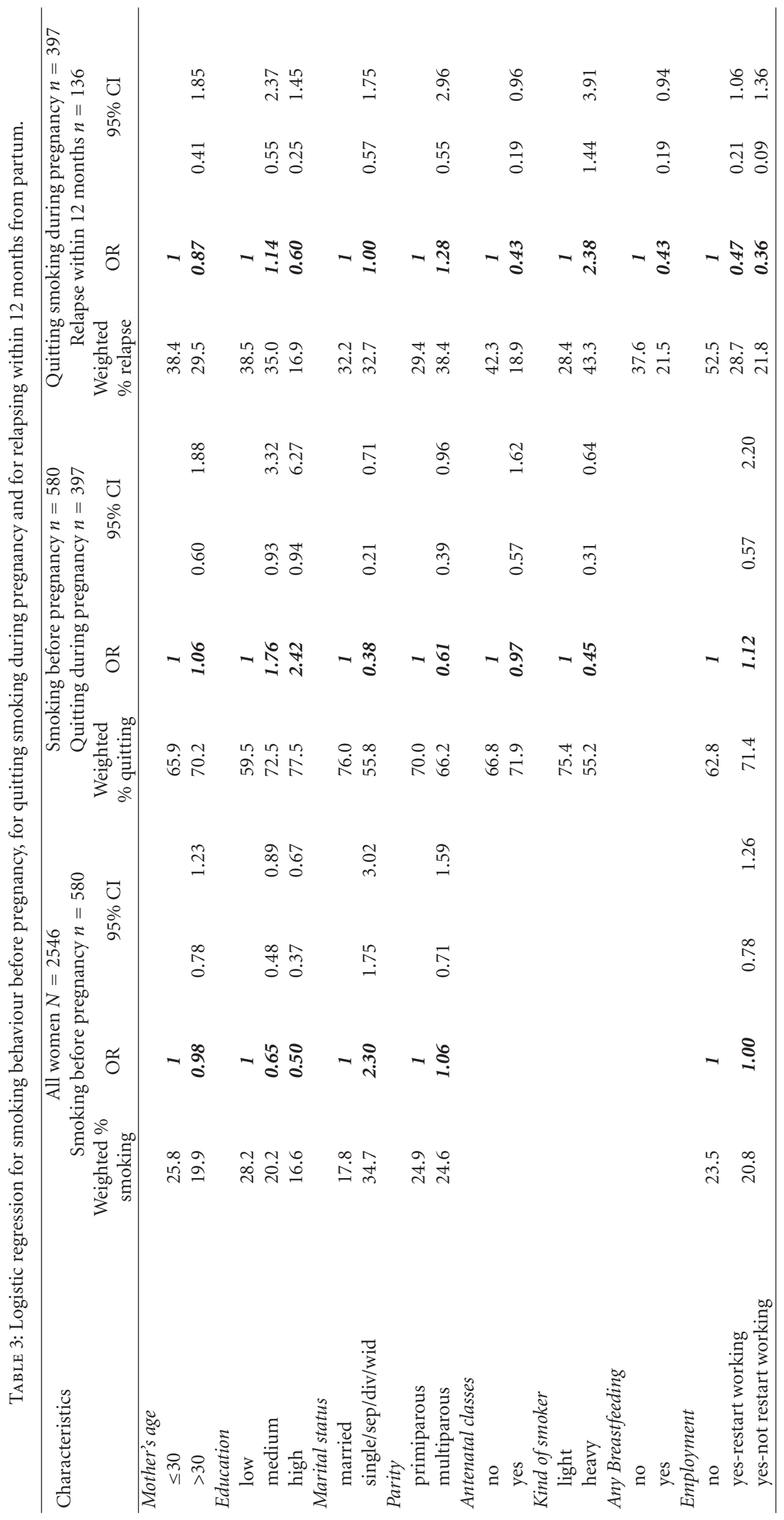


TABLE 4: Smoking relapse and the association with breastfeeding and maternal mood at each follow-up.

\begin{tabular}{|c|c|c|c|c|c|c|c|c|c|c|c|c|}
\hline \multirow[t]{3}{*}{ Characteristics } & \multirow{2}{*}{\multicolumn{4}{|c|}{$\begin{array}{l}\text { Follow-up } 3 \text { months } \\
\qquad n=461\end{array}$}} & \multirow{2}{*}{\multicolumn{4}{|c|}{$\begin{array}{l}\text { Follow-up } 6 \text { months } * \\
\qquad n=406\end{array}$}} & \multirow{2}{*}{\multicolumn{4}{|c|}{$\begin{array}{l}\text { Follow-up } 12 \text { months* } \\
\qquad n=415\end{array}$}} \\
\hline & & & & & & & & & & & & \\
\hline & $\begin{array}{l}\text { Weighted \% } \\
\text { relapse }\end{array}$ & $\mathrm{OR}^{* *}$ & \multicolumn{2}{|c|}{$95 \% \mathrm{CI}$} & $\begin{array}{l}\text { Weighted \% } \\
\text { relapse }\end{array}$ & $\mathrm{OR}^{* *}$ & \multicolumn{2}{|c|}{$95 \% \mathrm{CI}$} & $\begin{array}{l}\text { Weighted \% } \\
\text { relapse }\end{array}$ & $\mathrm{OR}^{* *}$ & \multicolumn{2}{|c|}{$95 \% \mathrm{CI}$} \\
\hline \multicolumn{2}{|l|}{ Breastfeeding*** } & & & & & & & & & & & \\
\hline No & 29.3 & 1 & & & 47.0 & 1 & & & 35.2 & 1 & & \\
\hline Yes & 8.4 & 0.23 & 0.09 & 0.58 & 14.3 & 0.22 & 0.11 & 0.44 & 19.6 & 0.41 & 0.18 & 0.92 \\
\hline \multicolumn{13}{|l|}{ Maternal mood } \\
\hline High & 16.8 & 1 & & & 28.3 & 1 & & & 34.6 & 1 & & \\
\hline Low & 25.0 & 1.73 & 1.21 & 2.47 & 41.9 & 1.73 & 1.03 & 2.90 & 26.9 & 0.71 & 0.46 & 1.10 \\
\hline
\end{tabular}

* Based on samples interviewed at each follow-up time.

** OR adjusted by all the variables indicated in Table 3 .

*** Breastfeeding: "full" at 3 months; "any" at 6 and 12 months.

and percentage of smoking women who continued to smoke in pregnancy $(31.3 \%)$ (ISTAT $29.3 \%$ ) were also found. The closeness of these results may indicate that our sample is representative. Nevertheless, the percentage of women who were lost at follow-up (28\%) are a limitation to the accuracy of the prevalence estimates after delivery. The respondents and nonrespondents were different with respect to sociodemographic characteristics, but not in smoking behaviour before and during pregnancy and in breastfeeding at birth. In addition, the multivariate analyses at 3- and 6-month follow-up were based on the total interviewed women at each follow-up point, $92 \%$ of the original sample at 3 months, and $91 \%$ at 6 months. Results did not differ if the multivariate analyses included only women who completed the follow-up, suggesting that those lost to follow-up may not introduce an important bias.

Another potential limitation of this study is that the measures of smoking status were self-reported and did not include biochemical validation of tobacco use. Studies have shown that self-reported measures of smoking status may be underestimated [25-27], and also, quitting smoking rates in pregnant women may be overestimated [28]. However, research has also shown that the accuracy of self-reported smoking status varies according to the settings in which the questions are asked. More specifically, intervieweradministered questionnaires, observational studies, reports by adults, and biochemical validation with cotinine plasma have been found to be associated with higher estimates of sensitivity and specificity [29]. Moreover, our previous study conducted with the same collection methods, on the association between smoking status and smoking intensity and low birth weight, showed results consistent with data reported in the literature, suggesting, indirectly, that the collected information was not strongly affected by these biases. Comparing our results with those of other Western countries, although the reference periods and the methodological approach may be different, smoking prevalences before pregnancy and during pregnancy are the lowest in Italy $(21.6 \%$ and $6.7 \%$, resp.) compared with the USA $22.4 \%$ and $14 \%[30,31]$, Japan $29.3 \%$ and $10.0 \%[18,32]$, France $35.9 \%$ and $21.8 \%$, [13], and UK 33.0\% (prevalence before or during pregnancy) and $17.0 \%$ [13]. Our prevalence results are also the lowest when compared with the samplebased estimates of other European countries: Belgium 22.4\% and $16.0 \%$, Bulgaria $33.2 \%$ and $7.0 \%$, Germany $38.7 \%$ and $16.9 \%$, Greece $30.0 \%$ and $11.7 \%$, Ireland $66.5 \%$ and $52.5 \%$, and Portugal 25.7\% and 14.3\%; [33]. The percentage decline in smoking prevalence during pregnancy was higher in Italy $(68.5 \%)$ than in all the other countries except Bulgaria (79\%). Nevertheless, tobacco use by pregnant women remains an important problem considering that the prevalence is far from the $2 \%$ goal in healthy people fixed in 2010 in USA [8]. Moreover, the 2009 project of the Italian Ministry of Health "Smoke-free mothers" establishes the prenatal smoking goal as less than $5 \%$ and a relapse prevention goal as less than $50 \%$ (on the base of a relapse rate estimate of about 70\%) [34]. Our results show that we are close to these goals, but a general increase in tobacco use in males and females, particularly among young people, was also noted in 2009, after a static period lasting 5 years $[15,16]$. Consequently, there is a real risk that smoking indicators among pregnant women could worsen.

Quitting and relapsing proportions at different followup times show that most change occurred within 6 months. Between 6 and 12 months there was a stabilization or a slow-down in the transitions regarding smoking behaviour. Heavy smokers are less likely to quit smoking and more likely to relapse compared with light smokers as found in other studies [31, 35-39]. Data from the literature indicate that the smoking relapse rate at 12 months postpartum is about 70$80 \%$ [40-43]. Our study shows a smoking relapse rate that is lower. In general, the results of this study, in comparison with reported values in other countries, show better smoking indicators at baseline and also lower relapsing rates at followup. Moreover, a sensitivity analysis was performed on women who quit during pregnancy. At each follow-up time, all women lost to follow-up were included in the analysis as they were relapsers. The results show estimates of relapsing rates of $26 \%$ within 3 months and $67-68 \%$ within 6 and 12 months, still lower in comparison with the literature. The strength of this study compared with others is that we have a measure of smoking behaviour collected by reinterviewing mothers at the three follow-up points, a procedure that is likely to reduce biases, in particular recall bias. 
The similar prevalences of smoking during pregnancy and at follow-up for breastfeeding women (about 5-7\%) suggest that these women are equally aware of the possible harmful effects for fetuses, during pregnancy, and for their children through breastfeeding, although the effects of nicotine on nursing infants are still largely unknown. It has been reported that maternal smoking may reduce the protective effect of breastfeeding (e.g., on respiratory allergy and infections, on Sudden Infant Death Syndrome) [44, 45 ] but it is not a contraindication to breastfeeding which remains beneficial and appropriate [46-48] and should be promoted also for those women who have difficulty with smoking cessation. However, health care professionals should advise all tobacco-using mothers to avoid smoking within the home and to smoke prior to breastfeeding.

In line with published literature $[21,39,49,50]$, factors associated with smoking behaviour changes during and after pregnancy in our study were education, marital status, parity, smoking intensity, and employment status at follow-up and breastfeeding. We also considered the participation in AC during pregnancy as a predictor factor and found a significant association with a reduction in smoking relapse risk. Breastfeeding, participation in AC, and smoking intensity before pregnancy represent the strongest factors associated with smoking relapse. We found that highly educated women were more likely to quit smoking and less likely to relapse. Participation in AC had no effect on quitting in pregnancy but reduced the smoking relapse risk. This may depend on the late beginning of these courses, which generally start around the 7th month of pregnancy. A possible effect of this factor on quitting smoking could be estimated if they started no more than 3-4 months after conception. Occupational status is also associated with smoking relapse risk. The risks are lower for women who were employed before pregnancy who stopped working during pregnancy and had not yet restarted working at 12 months. This may reflect the fact that women who plan not to return to work and those who do plan to return to work may have different perspectives about breastfeeding and tobacco use. It is also likely that stopping working for the period of maternity reduces work stress conditions that have been described in literature as risk factors for tobacco use [51].

Some studies have focused on the potential association between maternal mental health, such as low mood or stress, and quitting smoking during pregnancy or smoking relapse after delivery. The results are not exactly comparable given the different stress/mood measures used and, in fact, the need for a standard stress scale has been noted [21,38]. A relationship between postpartum psycological symptoms, breastfeeding and smoking relapse was also hypothesized $[21,27,52,53]$, although the nature of the relationship remains unclear. Our measure of maternal mood is an unspecific measure of distress that must not be confused with a postpartum depression marker. The strength of our study is in the availability of repeated measures of maternal mood, breastfeeding, and smoking behaviour at each followup time. Results show a significant independent effect of both maternal mood and breastfeeding on smoking relapse at 3 and 6 months even after adjusting for other potential confounders, while no effect of maternal mood was found at 12 months. This result might reflect the fact that the first months postpartum are a period in which mothers are subjected to a strong systematic source of stress, in particular sleep deprivation or sleep interruption. Previous studies on postpartum relapse and depressive symptoms or stress have reported contradictory results. Some studies found a statistically significant association between maternal mood and smoking relapse within 6 months, but only if breastfeeding was not included as a covariate in the analysis $[21,43]$. Others found an independent effect of breastfeeding and stress on smoking relapse, although stress was not statistically significant [18]. Since breastfeeding and maternal mood may be linked [52-54], interaction effects were also considered in post hoc regression analyses, but no significant results were found in our study.

The required information on the previous pregnancy for multiparous women gave us the opportunity of investigating retrospectively the association between breastfeeding a previous infant and smoking behaviour before the following pregnancy. The hypothesis we tested was that a prolonged breastfeeding might produce a reduction in smoking relapse, which is persistent in time. In our study, the values of risk parameters are not in contrast with this hypothesis but still it is not clear which causal mechanism may link breastfeeding with smoking behaviour. We could not determine if women breastfeeding the previous infant for more than 3 months were also less frequently smoking before the previous infant. In order to find some indications, we focused our attention on the present primiparous women assimilating them to the present multiparous women at the time of their previous infant. No statistically significant association was found between smoking behaviour before present pregnancy and breastfeeding at birth or at 3 months from partum, while a borderline but still not significant association was found with breastfeeding at 6 months. These results, even with all constrained assumptions and limits, might support our original hypothesis, which is consistent with the finding of several other studies that breastfeeding may protect against postpartum smoking relapse $[17-19,35$, 48].

In conclusion, our population-based study shows that women who are breastfeeding smoke less than not breastfeeding women. A strong association between tobacco use and breastfeeding was found even after controlling for other predictors. A low maternal mood increases the risk of smoking relapse within 6 months. This study also suggests that prolonged breastfeeding reduces the risk of smoking relapse and that this reduction may be persistent in time. Thus, interventions targeting breastfeeding promotion may indirectly support also smoking cessation, even in absence of specific interventions.

\section{References}

[1] European Commission Health and Consumer Protection Directorate General, "Survey on Tobacco and Analytical Report, 2009," http://ec.europa.eu/health/ph_determinants/ life_style/Tobacco/Documents/eb_253_en.pdf. Accessed 2011. 
[2] S. Murin, R. Rafii, and K. Bilello, "Smoking and Smoking Cessation in Pregnancy," Clinics in Chest Medicine, vol. 32, no. 1, pp. 75-91, 2011.

[3] U.S. Department of Health and Human Services, "How Tobacco smoke causes disease: the biology and behavioral basis for smoking-attributable disease: a report of the surgeon general. Atlanta, Ga: U.S. department of health and human services, centers for disease control and prevention, national center for chronic disease prevention and health promotion, office on smoking and health, 2010," http://www .surgeongeneral.gov/library/tobaccosmoke/report/full_report .pdf. Accessed 2011.

[4] W. Karmaus, A. L. Dobai, I. Ogbuanu, S. H. Arshard, S. Matthews, and S. Ewart, "Long-term effects of breastfeeding, maternal smoking during pregnancy, and recurrent lower respiratory tract infections on asthma in children," Journal of Asthma, vol. 45, no. 8, pp. 688-695, 2008.

[5] S. Cnattingius, "The epidemiology of smoking during pregnancy: smoking prevalence, maternal characteristics, and pregnancy outcomes," Nicotine and Tobacco Research, vol. 6, no. 2, pp. S125-S140, 2004.

[6] A. Einarson and S. Riordan, "Smoking in pregnancy and lactation: a review of risks and cessation strategies," European Journal of Clinical Pharmacology, vol. 65, no. 4, pp. 325-330, 2009.

[7] C. Obel, J. Olsen, T. B. Henriksen et al., "Is maternal smoking during pregnancy a risk factor for Hyperkinetic disorder? Findings from a sibling design," International Journal of Epidemiology, vol. 40, no. 2, pp. 338-345, 2011.

[8] Office of Disease Prevention and Health Promotion and U.S. Department of Health and Human Services, "Healthy People 2010 Objectives," http://www.healthypeople.gov. Accessed 2011.

[9] B. Al-Sahab, M. Saqib, G. Hauser, and H. Tamim, "Prevalence of smoking during pregnancy and associated risk factors among Canadian women: a national survey," BMC Pregnancy and Childbirth, vol. 10, article 24, 2010.

[10] K. Egebjerg Jensen, A. Jensen, B. Nøhr, and S. Krüger Kjær, "Do pregnant women still smoke? A study of smoking patterns among 261,029 primiparous women in Denmark 1997-2005," Acta Obstetricia et Gynecologica Scandinavica, vol. 87, no. 7, pp. 760-767, 2008.

[11] P. Laws, S. Abeywardana, J. Walker, and E. A. Sullivan, Australia's Mothers and Babies 2005, AIHW, Canberra, Australia, 2007.

[12] Y. Kaneita, S. Tomofumi, S. Takemura et al., "Prevalence of smoking and associated factors among pregnant women in Japan," Preventive Medicine, vol. 45, no. 1, pp. 15-20, 2007.

[13] "EURO-PERISTAT. European Perinatal Health Report by the EURO-PERISTAT project in collaboration with SCPE, EUROCAT \& EURONEOSTAT, 2008," http://www.europeristat.com. Accessed 2011.

[14] Istituto Nazionale di Statistica (ISTAT), Indagine Multiscopo sulle Famiglie "Condizioni di Salute e Ricorso ai Servizi Sanitari"-2005, ISTAT, Rome, Italy, 2007.

[15] Istituto Nazionale di Statistica (ISTAT), "Annuario "Sanità e salute" Rome: ISTAT; 2009," http://www.istat.it/dati/catalogo/ 20091120_00/contenuti.html. Accessed 2011.

[16] "Indagine annuale Doxa/Iss-Ofad 2009. Istituto Superiore di Sanità; 2009," http://www.iss.it/binary/ofad/cont/Zuccaro_ Milano_2009_cartella_stampa.pdf. Accessed 2011.

[17] A. R. Letourneau, B. Sonja, C. M. Mazure, S. S. O’Malley, D. James, and E. R. Colson, "Timing and predictors of postpartum return to smoking in a group of inner-city women: an exploratory pilot study," Birth, vol. 34, no. 3, pp. 245-252, 2007.

[18] A. Kaneko, Y. Kaneita, E. Yokoyama et al., "Smoking trends before, during, and after pregnancy among women and their spouses," Pediatrics International, vol. 50, no. 3, pp. 367-375, 2008.

[19] K. I. DiSantis, B. N. Collins, and A. C. McCoy, "Associations among breastfeeding, smoking relapse, and prenatal factors in a brief postpartum smoking intervention," Acta Obstetricia et Gynecologica Scandinavica, vol. 89, no. 4, pp. 582-586, 2010.

[20] B. L. Horta, M. S. Kramer, and R. W. Platt, "Maternal smoking and the risk of early weaning: a meta-analysis," American Journal of Public Health, vol. 91, no. 2, pp. 304-307, 2001.

[21] M. E. Gyllstrom, W. L. Hellerstedt, and D. Hennrikus, "The Association of Maternal Mental Health with Prenatal Smoking Cessation and Postpartum Relapse in a Population-Based Sample," Maternal and Child Health Journal. In press.

[22] T. M. Higgins, S. T. Higgins, S. H. Heil et al., "Effects of cigarette smoking cessation on breastfeeding duration," Nicotine and Tobacco Research, vol. 12, no. 5, pp. 483-488, 2010.

[23] P. A. Ratner, J. L. Johnson, and J. L. Bottorff, "Smoking relapse and early weaning among postpartum women: is there an association?" Birth, vol. 26, no. 2, pp. 76-82, 1999.

[24] M. Grandolfo, L. Lauria, and A. Lamberti, “ Il percorso nascita: promozione e valutazione della qualità dei modelli operativi. Principali risultati dell'indagine sul percorso nascita. Presentato al Convegno "Il percorso nascita nella prospettiva del Progetto Obiettivo Materno Infantile -POMI"; Istituto Superiore di Sanità, Roma," http://www.epicentro.iss .it/problemi/percorso-nascita/PDF_Convegno_28-Apr-2010/ Lauria_Principali\%20Risultati_.pdf, 2010.

[25] P. M. Dietz, D. Homa, L. J. England et al., "Estimates of nondisclosure of cigarette smoking among pregnant and nonpregnant women of reproductive age in the United States," American Journal of Epidemiology, vol. 173, no. 3, pp. 355-359, 2011.

[26] D. Shipton, D. M. Tappin, T. Vadiveloo, J. A. Crossley, D. A. Aitken, and J. Chalmers, "Reliability of self reported smoking status by pregnant women for estimating smoking prevalence: a retrospective, cross sectional study," BMJ, vol. 339, p. b4347, 2009.

[27] W. Hannöver, J. R. Thyrian, A. Ebner et al., "Smoking during pregnancy and postpartum: smoking rates and intention to quit smoking or resume after pregnancy," Journal of Women's Health, vol. 17, no. 4, pp. 631-640, 2008.

[28] L. J. England, A. Grauman, C. Qian et al., "Misclassification of maternal smoking status and its effects on an epidemiologic study of pregnancy outcomes," Nicotine and Tobacco Research, vol. 9, no. 10, pp. 1005-1013, 2007.

[29] D. L. Patrick, A. Cheadle, D. C. Thompson, P. Diehr, T. Koepsell, and S. Kinne, "The validity of self-reported smoking: a review and meta-analysis," American Journal of Public Health, vol. 84, no. 7, pp. 1086-1093, 1994.

[30] Centers for Disease Control and Prevention (CDCP), "Smoking prevalence among women of reproductive age-United States, 2006," Morbidity and Mortality Weekly Report, vol. 57, pp. 849-852, 2008.

[31] V. T. Tong, J. R. Jones, P. M. Dietz, D. D'Angelo, and J. M. Bombard, "Trends in smoking before, during, and after pregnancy_pregnancy risk assessment monitoring system (PRAMS), United States, 31 sites, 2000-2005," MMWR Surveillance Summaries, vol. 58, no. SS-4, pp. 1-29, 2009.

[32] Surveys on the Growth of Infants and Preschool Children Japan, Ministry of Health, Labour and Welfare, Tokyo, Japan, 2000. 
[33] K. Giersiepen, B. Janssen, L. Tsoneva-Pentcheva et al., "Euro-scip-III-Survey: an International Comparison of Smoking Prevalence in Pregnant Women. Bremen Institute for Prevention Research and Social Medicine (BIPS), Bremen, Germany, 2006," http://www.womenofthenorthwest .net/newsletters/Bips\%20Pregnancy\%20and\%20Smoking\% 20Research\%20Report.pdf. Accessed 2011.

[34] Ministero della Salute, "Attività per la prevenzione del tabagismo: rapporto 2009. Ministero della Salute; 2009," http://www.salute.gov.it/imgs/C_17_newsAree_844_listaFile_ itemName_0_file.pdf. Accessed 2011.

[35] L. T. Martin, M. McNamara, A. Milot, M. Bloch, E. C. Hair, and T. Halle, "Correlates of smoking before, during, and after pregnancy," American Journal of Health Behavior, vol. 32, no. 3, pp. 272-282, 2008.

[36] L. Solomon and V. Quinn, "Spontaneous quitting: selfinitiated smoking cessation in early pregnancy," Nicotine and Tobacco Research, vol. 6, no. 2, pp. S203-S216, 2004.

[37] S. M. Yu, C. H. Park, and R. H. Schwalberg, "Factors associated with smoking cessation among U.S. pregnant women," Maternal and Child Health Journal, vol. 6, no. 2, pp. 89-97, 2002.

[38] T. J. Linares Scott, S. H. Heil, S. T. Higgins, G. J. Badger, and I. M. Bernstein, "Depressive symptoms predict smoking status among pregnant women," Addictive Behaviors, vol. 34, no. 8, pp. 705-708, 2009.

[39] J. Liu, K. D. Rosenberg, and A. P. Sandoval, "Breastfeeding duration and perinatal cigarette smoking in a populationbased cohort," American Journal of Public Health, vol. 96, no. 2, pp. 309-314, 2006.

[40] "Center Women and smoking: a report of the Surgeon General. Executive summary," MMWR Recommendations and Reports, vol. 51, no. RR-12, pp. 1-13, 2002.

[41] R. S. Kahn, L. Certain, and R. C. Whitaker, "A reexamination of smoking before, during, and after pregnancy," American Journal of Public Health, vol. 92, no. 11, pp. 1801-1808, 2002.

[42] G. J. Colman and T. Joyce, "Trends in smoking before, during, and after pregnancy in ten states," American Journal of Preventive Medicine, vol. 24, no. 1, pp. 29-35, 2003.

[43] D. Ershoff, T. H. Ashford, and R. Goldenberg, "Helping pregnant women quit smoking: an overview," Nicotine and Tobacco Research, vol. 6, no. 2, pp. S101-S105, 2004.

[44] H. S. Klonoff-Cohen, S. L. Edelstein, E. Schneider Lefkowitz et al., "The effect of passive smoking and tobacco exposure through breast milk on SIDS," Journal of the American Medical Association, vol. 273, no. 10, pp. 796-798, 1995.

[45] H. T. Guedes and L. S. Souza, "Exposure to maternal smoking in the first year of life interferes in breast-feeding protective effect against the onset of respiratory allergy from birth to 5 yr," Pediatric Allergy and Immunology, vol. 20, no. 1, pp. 3034, 2009.

[46] American Academy of Pediatrics Committee on Drugs, "Transfer of drugs and other chemicals into human milk," Pediatrics, vol. 108, pp. 776-789, 2001.

[47] L. M. Gartner, J. Morton, R. A. Lawrence et al., "Breastfeeding and the use of human milk," Pediatrics, vol. 115, pp. 496-506, 2005.

[48] J. G. Dorea, "Maternal smoking and infant feeding: breastfeeding is better and safer," Maternal and Child Health Journal, vol. 11, no. 3, pp. 287-291, 2007.

[49] D. E. Kendzor, M. S. Businelle, T. J. Costello et al., "Breast feeding is associated with postpartum smoking abstinence among women who quit smoking due to pregnancy," Nicotine and Tobacco Research, vol. 12, no. 10, pp. 983-988, 2010.
[50] T. M. Weiser, M. Lin, V. Garikapaty, R. W. Feyerharm, D. M. Bensyl, and B. P. Zhu, "Association of maternal smoking status with breastfeeding practices: Missouri, 2005," Pediatrics, vol. 124, no. 6, pp. 1603-1610, 2009.

[51] A. Kouvonen, M. Kivimäki, M. Virtanen, J. Pentti, and J. Vahtera, "Work stress, smoking status, and smoking intensity: an observational study of 46190 employees," Journal of Epidemiology and Community Health, vol. 59, no. 1, pp. 6369, 2005.

[52] K. H. Gross, C. S. Wells, A. Radigan-Garcia, and P. M. Dietz, "Correlates of self-reports of being very depressed in the months after delivery: results from the Pregnancy Risk Assessment Monitoring System," Maternal and Child Health Journal, vol. 6, no. 4, pp. 247-253, 2002.

[53] C. L. Dennis and K. McQueen, "The relationship between infant-feeding outcomes and postpartum depression: a qualitative systematic review," Pediatrics, vol. 123, no. 4, pp. e736e751, 2009.

[54] B. J. Morasco, E. A. Dornelas, E. H. Fischer, C. Oncken, and H. A. Lando, "Spontaneous smoking cessation during pregnancy among ethnic minority women: a preliminary investigation," Addictive Behaviors, vol. 31, no. 2, pp. 203-210, 2006. 


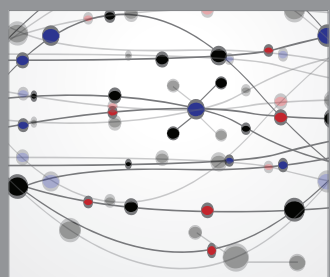

The Scientific World Journal
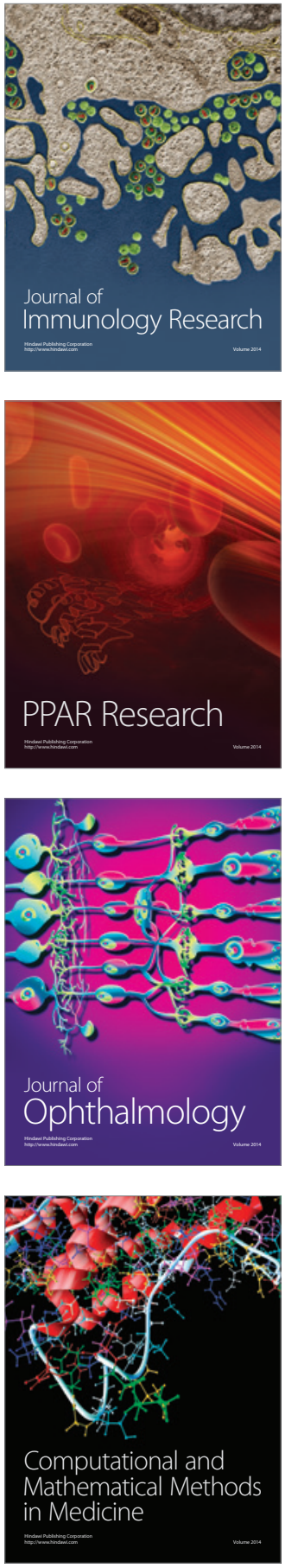

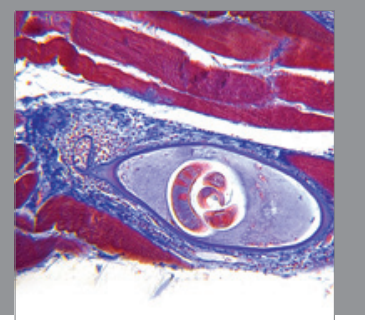

Gastroenterology

Research and Practice
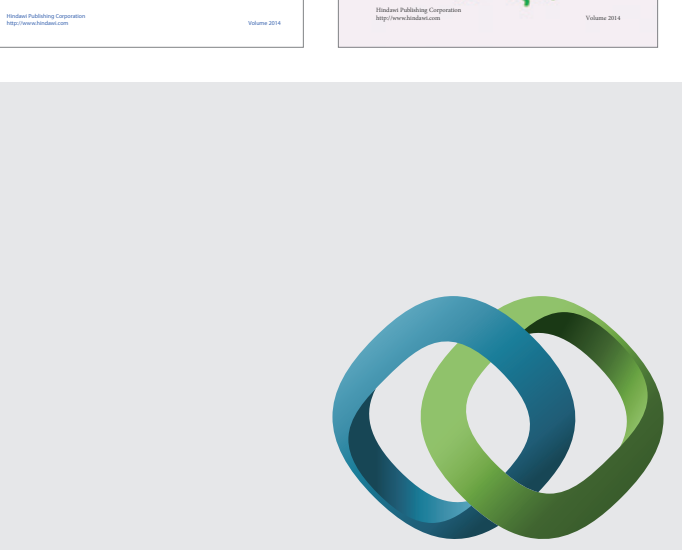

\section{Hindawi}

Submit your manuscripts at

http://www.hindawi.com
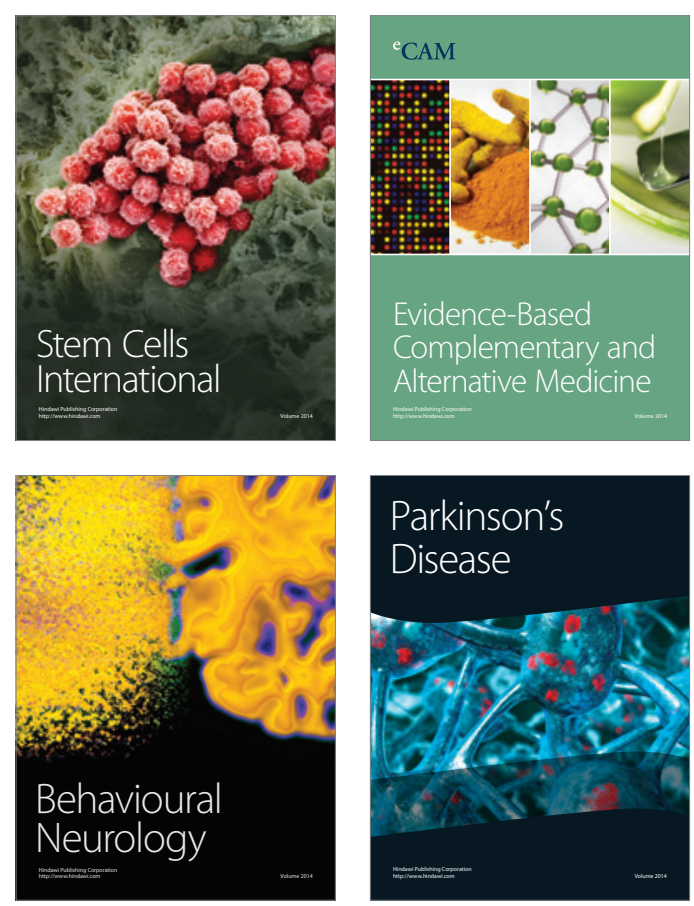

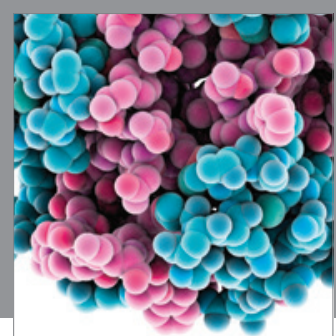

Journal of
Diabetes Research

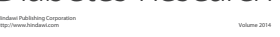

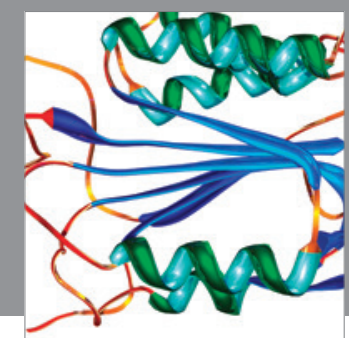

Disease Markers
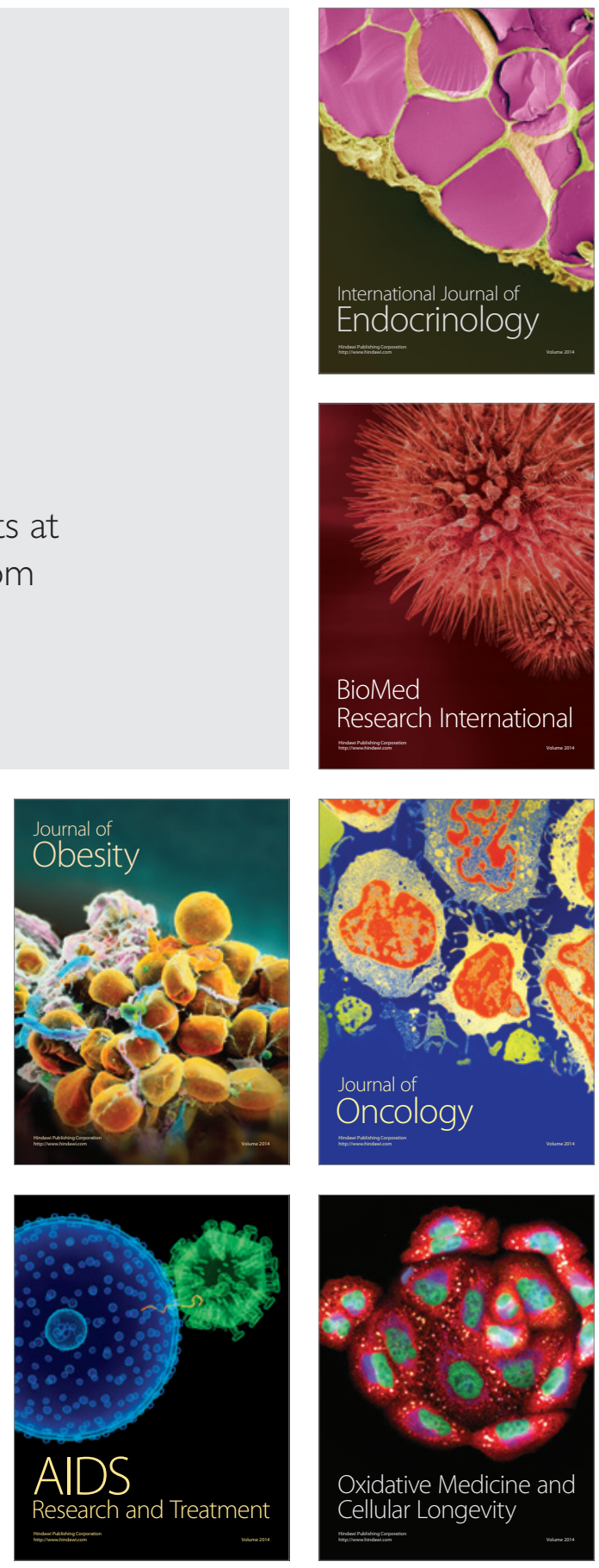